To Appear in the Astrophysical Journal

Preprint typeset using $\mathrm{L}_{\mathrm{A}} \mathrm{X}$ style emulateapj v. 01/23/15

\title{
IS MOLECULAR CLOUD TURBULENCE DRIVEN BY EXTERNAL SUPERNOVA EXPLOSIONS?
}

\author{
DANIEL SEIFRIED* \\ I. Physikalisches Institut, Universität zu Köln, Zülpicher Str. 77, 50937 Köln, Germany \\ SteFANIE WALCH \\ I. Physikalisches Institut, Universität zu Köln, Zülpicher Str. 77, 50937 Köln, Germany \\ Sebastian Haid \\ I. Physikalisches Institut, Universität zu Köln, Zülpicher Str. 77, 50937 Köln, Germany \\ PHILIPP GIRICHIDIS \\ Leibniz-Institut fur Astrophysik Potsdam (AIP), An der Sternwarte 16, 14482 Potsdam, Germany \\ Heidelberg Institute for Theoretical Studies, Schloss-Wolfsbrunnenweg 35, 69118 Heidelberg, Germany \\ THORSTEN NAAB \\ Max-Planck-Institut für Astrophysik, Karl-Schwarzschild-Str. 1, 85741 Garching, Germany \\ (Received; Revised; Accepted) \\ To Appear in the Astrophysical Journal
}

\begin{abstract}
We present high-resolution $(\sim 0.1 \mathrm{pc})$, hydrodynamical and magnetohydrodynamical simulations to investigate whether the observed level of molecular cloud (MC) turbulence can be generated and maintained by external supernova (SN) explosions. The MCs are formed self-consistently within their large-scale galactic environment following the non-equilibrium formation of $\mathrm{H}_{2}$ and $\mathrm{CO}$ including (self-) shielding and important heating and cooling processes. The MCs inherit their initial level of turbulence from the diffuse ISM, where turbulence is injected by SN explosions. However, by systematically exploring the effect of individual SNe going off outside the clouds, we show that at later stages the importance of SN-driven turbulence is decreased significantly. This holds for different MC masses as well as for MCs with and without magnetic fields. The SN impact also decreases rapidly with larger distances. Nearby $\mathrm{SNe}(d \sim 25 \mathrm{pc})$ boost the turbulent velocity dispersions of the MC by up to 70 per cent (up to a few $\mathrm{km} \mathrm{s}^{-1}$ ). For $d>50 \mathrm{pc}$, however, their impact decreases fast with increasing $d$ and is almost negligible. For all probed distances the gain in velocity dispersion decays rapidly within a few $100 \mathrm{kyr}$. This is significantly shorter than the average timescale for an MC to be hit by a nearby SN under solar neighbourhood conditions $(\sim 2 \mathrm{Myr})$. Hence, at these conditions $\mathrm{SNe}$ are not able to sustain the observed level of MC turbulence. However, in environments with high gas surface densities and SN rates ,like the Central Molecular Zone, observed elevated MC dispersions could be triggered by external SNe.

Keywords: astrochemistry — magnetohydrodynamics (MHD) — methods: numerical — ISM: clouds — ISM: kinematics and dynamics — ISM: supernova remnants
\end{abstract}

\section{INTRODUCTION}

Molecular clouds (MCs) are observed to have large non-thermal line widths (e.g. Larson 1981; Solomon et al. 1987) indicating supersonic turbulence. This turbulence is present at early as well as later stages when star formation has already set in (e.g Williams et al. 1994; Hirota et al. 2011). However, supersonic turbulence is expected to decay in a turbulent crossing time (e.g. Mac Low et al. 1998 Stone et al. 1998), which is typically shorter (a few Myr) than the cloud lifetime ( 10 Myr; see e.g. Dobbs et al. 2014). Hence, the observed level of turbulence needs to be driven by either internal stellar feedback (see e.g. Elmegreen \& Scalo 2004; Mac Low \& Klessen 2004, Ballesteros-Paredes et al. 2007, for reviews), local gravitational collapse (Ibáñez-Mejía et al. 2016, 2017), gas

*seifried@ph1.uni-koeln.de accretion onto the cloud (Klessen \& Hennebelle 2010 Goldbaum et al. 2011), or supernovae (SNe) located outside the cloud (Padoan et al. 2016).

$\mathrm{SNe}$ are considered as one of the main drivers of interstellar turbulence (e.g. Elmegreen \& Scalo 2004, Mac Low \& Klessen 2004; Walch \& Naab 2015; Naab \& Ostriker 2017) and are involved in the formation of MCs e.g. by sweeping up material (Koyama \& Inutsuka 2000). Although there are observations of the interaction of SN remnants with MCs at infrared and $X$ $/ \gamma$-ray wavelengths (e.g. Neufeld et al. 2007; Hewitt \& Yusef-Zadeh 2009: Tang \& Chevalier 2014), it remains unclear whether SNe can maintain the internal velocity dispersion of MCs after the cloud has formed. To investigate this, realistic MC formation models including their surrounding galactic environment, through which the SN shock has to travel before it hits the clouds, 
are required. Such studies are numerically demanding and recent works by Ibáñez-Mejía et al. (2016, 2017) and Padoan et al. (2016) come to different conclusions. Padoan et al. (2016) argue that external SNe alone can maintain the observed level of turbulence, whereas Ibáñez-Mejía et al. (2016, 2017) suggest that gravity rather than SNe causes the observed turbulent velocity dispersion.

Here, we present a novel study, which systematically explores the effect of SNe at varying distances on selfconsistently formed MCs embedded in their galactic environment (Seifried et al. 2017). The simulations include a chemical network with associated self-consistent cooling and heating processes in combination with a high spatial resolution of $0.12 \mathrm{pc}$

\section{NUMERICAL METHODS}

The simulations presented here make use of the zoomin technique presented in Seifried et al. (2017) for MC formation within the SILCC project (see Walch et al. 2015. Girichidis et al. 2016, for details). The simulations are performed with the adaptive mesh refinement code FLASH 4.3 (Fryxell et al. 2000, Dubey et al. 2008) using a magnetohydrodynamics solver which guarantees positive entropy and density (Bouchut et al. 2007; Waagan 2009). We model the chemical evolution of the interstellar medium (ISM) using a simplified chemical network for $\mathrm{H}^{+}, \mathrm{H}, \mathrm{H}_{2}, \mathrm{C}^{+}, \mathrm{CO}, \mathrm{e}^{-}$, and $\mathrm{O}$ (Nelson \& Langer 1997; Glover \& Mac Low 2007, Glover et al. 2010), which also follows the thermal evolution of the gas including the most relevant heating and cooling processes. The shielding of the interstellar radiation field $\left(G_{0}=1.7\right.$; Habing 1968 Draine 1978) is calculated according to the surrounding column densities of total gas, $\mathrm{H}_{2}$, and $\mathrm{CO}$ via the TREeCoL algorithm (Clark et al. 2012; Walch et al. 2015. Wünsch et al. 2017). We solve the Poisson equation for self-gravity with a tree-based method (Wünsch et al. 2017) and include a background potential from the preexisting stellar component in the galactic disc, modeled as an isothermal sheet with $\Sigma_{\text {star }}=30 \mathrm{M}_{\odot} \mathrm{pc}^{-2}$ and a scale height of $100 \mathrm{pc}$.

Our setup represents a small section of a galactic disc with solar neighborhood properties and a size of 500 $\mathrm{pc} \times 500 \mathrm{pc} \times \pm 5 \mathrm{kpc}$. The gas surface density is $\Sigma_{\text {gas }}=10 \mathrm{M}_{\odot} \mathrm{pc}^{-2}$ and the initial vertical distribution of the gas has a Gaussian profile with a scale height of $30 \mathrm{pc}$ and a midplane density of $9 \times 10^{-24} \mathrm{~g} \mathrm{~cm}^{-3}$. The gas near the disc midplane has an initial temperature of $4500 \mathrm{~K}$ and consists of atomic hydrogen and $\mathrm{C}^{+}$.

Up to $t_{0}=11.9 \mathrm{Myr}$, we drive turbulence in the disc with SNe, which are randomly placed in the midplane and have a Gaussian distribution with a scale height of $50 \mathrm{pc}$ in the vertical direction. The SN rate is constant at $15 \mathrm{SNe} \mathrm{Myr}^{-1}$, corresponding to the Kennicutt-Schmidt star formation rate surface density for $\Sigma_{\text {gas }}=10 \mathrm{M}_{\odot} \mathrm{pc}^{-2}$ Kennicutt 1998). For a single SN we inject $10^{51}$ erg in the form of thermal energy if the Sedov-Taylor radius is resolved with at least 4 cells. Otherwise, we heat the gas within the injection region to $10^{4} \mathrm{~K}$ and inject the momentum, which the swept-up shell has gained at the end of the pressure-driven snowplow phase (see Blondin et al. 1998, and Gatto et al.|2015 for details).

The base grid resolution is $3.9 \mathrm{pc}$ up to $t_{0}$. At $t_{0}$ we

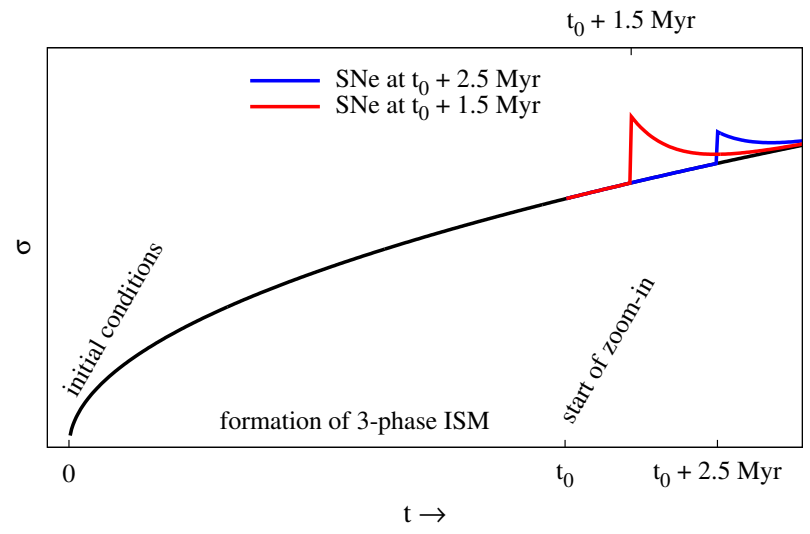

Figure 1. Schematic view of the different phases in the simulations showing the initial phase up to $t_{0}$ for the evolution of the three-phase medium and the different times $t_{\mathrm{SN}}=t_{0}+1.5 \mathrm{Myr}$ and $t_{0}+2.5 \mathrm{Myr}$ at which SNe are inserted for the parameter study. For the runs with magnetic fields (Section 3.4 , we insert $\mathrm{SNe}$ at $t=t_{0, \mathrm{mag}}+1.5$ and $t_{0, \mathrm{mag}}+2.5 \mathrm{Myr}$.

stop further SN explosions. We choose two regions in which MCs - henceforth denoted as MC1 and MC2 - are about to form and we continue the simulation for another 1.5 Myr over which we progressively increase the spatial resolution $\mathrm{d} x$ in these two regions from $3.9 \mathrm{pc}$ to $0.12 \mathrm{pc}$ (Seifried et al. 2017, Table 2).

In order to estimate the time required to develop a realistic three-phase ISM from the quiescent initial conditions, we assume a mean expansion speed of our SN remnants of $\sim 20-30 \mathrm{~km} \mathrm{~s}^{-1}\left(\simeq 20-30 \mathrm{pc} \mathrm{Myr}^{-1}\right)$ and a mean SN age of $t_{0} / 2=6 \mathrm{Myr}$. Hence, each SN remnant would cover on average a spherical region with about $240-360 \mathrm{pc}$ in diameter. Given the SN rate of $15 \mathrm{SNe} \mathrm{Myr}^{-1}, 180 \mathrm{SNe}$ are inserted up to $t_{0}$. Comparing the area which would be covered by all 180 SNe to the area of the simulated part of the disk $(500 \mathrm{pc} \times 500 \mathrm{pc})$ gives us the typical number of how often each point in the disk is hit by a SN shock. With the numbers above we find that up to $t_{0}$ each point in the disk midplane is affected on average by $\sim 50 \mathrm{SNe}$. Although this is a rather crude estimate, it shows that a time of $\sim 12$ Myr is sufficient to develop a self-consistent, turbulent three-phase ISM.

Shortly after the clouds are fully refined, at $t_{\mathrm{SN}}=t_{0}+1.53 \mathrm{Myr}$ we explode single SNe at different distances of $d=25,50,62.5$, or 75 pc from the center-ofmass of either cloud along the $\pm x-, \pm y$-, or $\pm z$-direction. In addition, we also investigate the impact of $\mathrm{SNe}$ going off $\sim 1$ Myr later at $t_{\mathrm{SN}}=t_{0}+2.5 \mathrm{Myr}$ (Section 3.5). A schematic overview of the different times in our simulations is shown in Figure 1 .

Furthermore, in Section 3.4 we investigate the influence of magnetic fields on our results. For this, we zoom-in on 2 clouds denoted as MC3 and MC4 at $t_{0, \text { mag }}=16 \mathrm{Myr}$ in a comparable simulation with magnetic fields. In total, we consider 132 different simulations including SNe (see Table 1), thus giving us statistically reliable results.

\section{RESULTS}

\subsection{Column density evolution}

In Seifried et al. (2017) we have investigated the formation and early evolution of MC1 and MC2 for $5 \mathrm{Myr}$, i.e. up to $t_{0}+5 \mathrm{Myr}$, without any further $\mathrm{SNe}$ in the 


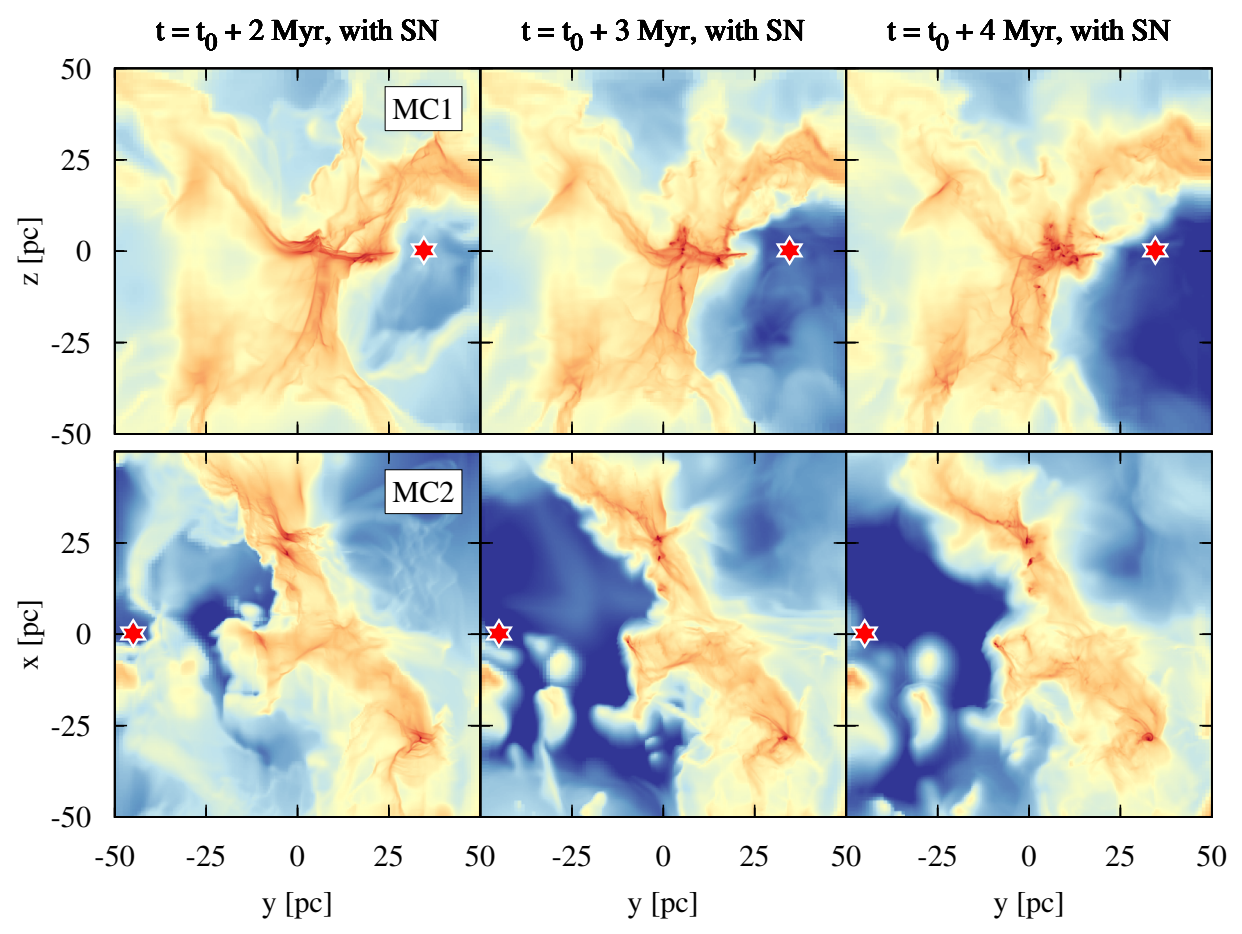

$\mathrm{t}=\mathrm{t}_{0}+4 \mathrm{Myr}$, no SN $\log \left(\Sigma\left[\mathrm{g} \mathrm{cm}^{-2}\right]\right)$

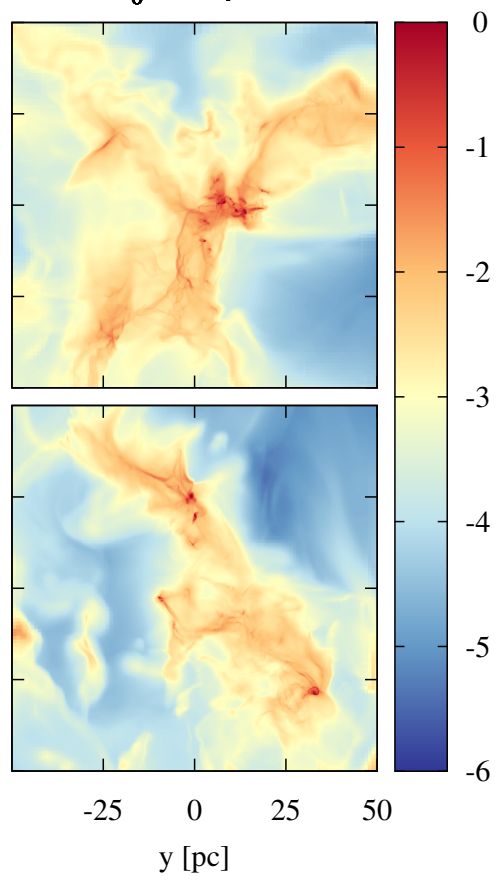

Figure 2. Time evolution $\left(t=t_{0}+2 \mathrm{Myr}\right.$ to $\left.t_{0}+4 \mathrm{Myr}\right)$ of the column density of MC1 (top row) and MC2 (bottom row) as they are hit by a nearby SN (red star). For comparison the right panels show the reference run with no SN at $t_{0}+4$ Myr. The comparable structure of the clouds with and without a SN indicates that the SN mainly affects the low-density environment.

Table 1

Overview of the performed simulations showing the cloud name, the time $t_{0}$, at which we start to zoom-in, the distance $d$ and time $t_{\mathrm{SN}}$ the SN goes off, and whether magnetic fields are included.

\begin{tabular}{ccccc}
\hline cloud & $d[\mathrm{pc}]$ & $t_{0}[\mathrm{Myr}]$ & $t_{\mathrm{SN}}[\mathrm{Myr}]$ & B-field \\
\hline MC1 & $25,50,62.5,75$ & 11.9 & 13.4 & No \\
MC2 & $25,50,62.5,75$ & 11.9 & 13.4 & No \\
MC1 & 25,50 & 11.9 & 14.4 & No \\
MC2 & 25,50 & 11.9 & 14.4 & No \\
\hline MC3 & $25,50,62.5$ & 16.0 & 17.5 & Yes \\
MC4 & $25,50,62.5$ & 16.0 & 17.5 & Yes \\
MC3 & 25,50 & 16.0 & 18.5 & Yes \\
MC4 & 25,50 & 16.0 & 18.5 & Yes \\
\hline
\end{tabular}

simulation domain. These simulations serve as the reference simulations indicated with the subscript "no SN". The clouds harbor increasing amounts of molecular gas and develop a strongly fragmented sub-structure (right panels of Figure 22.

The left panels show the time evolution of the column density of MC1 (top row) and MC2 (bottom row) which are exposed to a SN at a distance of 25 and $50 \mathrm{pc}$, respectively. The SN bubbles expand asymmetrically and seem not to be able to significantly affect the central, high-density regions. A qualitatively similar behavior is found for all other simulations.

\subsection{Time evolution}

In Figure 3 we show the time evolution of the mass and velocity dispersion of the two non-magnetic clouds. We define the mass $M$ of the cloud as the mass of all gas with particle densities $n \geq 100 \mathrm{~cm}^{-3}$ (assuming a mean molecular weight of $\mu=2.3$ ). The velocity dispersion $\sigma$ of this mass is then defined as the mass-weighted root-
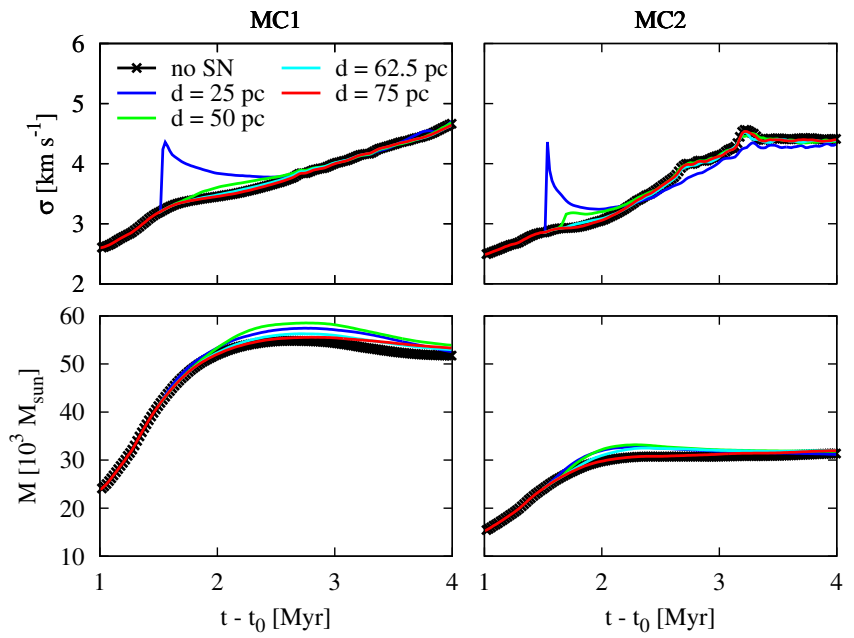

Figure 3. Time evolution of the velocity dispersion (top row) and the mass (bottom row) of the non-magnetic clouds MC1 (left) and MC2 (right) calculated for gas with densities $\geq 100 \mathrm{~cm}^{-3}$. The black line shows $\sigma_{\text {no SN }}$ and $M_{\text {no SN }}$ from the reference runs, the remaining lines show $\sigma_{\mathrm{SN}}$ and $M_{\mathrm{SN}}$ for SNe exploding at distances of $25,50,62.5$, and $75 \mathrm{pc}$ along the -y-direction.

mean-square velocity with respect to the center-of-mass $(\mathrm{COM})$ velocity of the cloud, $\vec{v}_{\mathrm{COM}}$, i.e.

$$
\sigma=\sqrt{\frac{\sum\left(\mathrm{d} m \times\left(\vec{v}-\vec{v}_{\mathrm{COM}}\right)^{2}\right)}{\Sigma \mathrm{d} m}},
$$

where $\mathrm{d} m$ and $\vec{v}$ are the mass and velocity of an individual cell, respectively, and we sum over all cells with $n \geq 100 \mathrm{~cm}^{-3}$.

For the unperturbed clouds, the masses $M_{\text {noSN }}$ increase over about $2 \mathrm{Myr}$ and afterward remain roughly constant, with values around $5 \times 10^{4} \mathrm{M}_{\odot}$ and $3 \times$ 

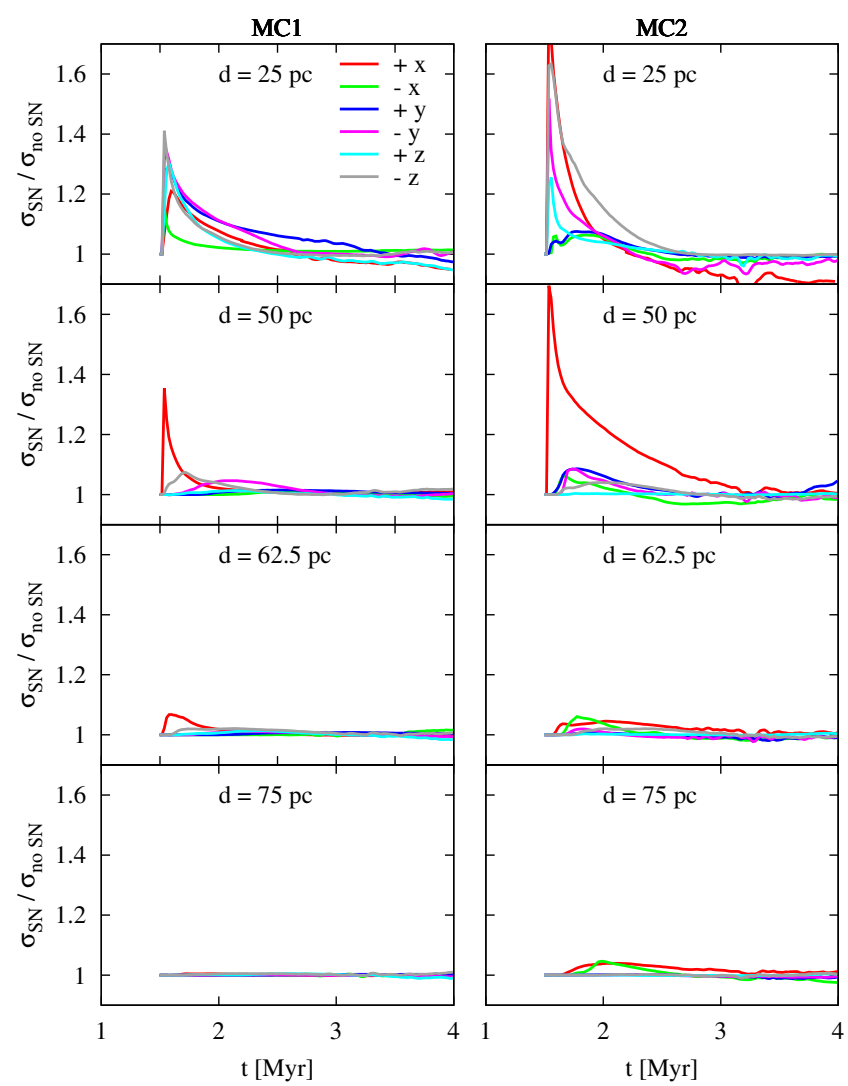

Figure 4. Relative change, $\sigma_{\mathrm{SN}} / \sigma_{\mathrm{no} S \mathrm{SN}}$, for SNe going off at increasing distances (from top to bottom) along the $\pm x-, \pm y$-, and $\pm z$-directions for $\mathrm{MC} 1$ (left) and $\mathrm{MC} 2$ (right) calculated for gas with densities $\geq 100 \mathrm{~cm}^{-3}$. At distances up to $50 \mathrm{pc}, \sigma$ is temporarily (for $\sim 100 \mathrm{kyr}$ ) enhanced up to $70 \%$ compared with the respective reference simulation. At larger distances the $\mathrm{SNe}$ have very little effect.

$10^{4} \mathrm{M}_{\odot}$ for $\mathrm{MC} 1$ and $\mathrm{MC} 2$, respectively (bottom row of Figure 3, black line with crosses). The velocity dispersion of the unperturbed clouds $\sigma_{\text {no SN }}$ (top row) increases continuously for MC1, whereas for MC2 around $t \simeq t_{0}+3 \mathrm{Myr}$ it levels off at $\sigma_{\mathrm{noSN}} \simeq 4.5 \mathrm{~km} \mathrm{~s}^{-1}$. We emphasize that both, the derived masses and velocity dispersions indicate that the chosen clouds are representative of typical MCs in the Milky Way, for example Miville-Deschênes et al. (2017) found a median cloud mass of $4 \times 10^{4} \mathrm{M}_{\odot}$ (but see also e.g. Solomon et al. 1987, Elmegreen \& Falgarone 1996, Heyer et al. 2001; Roman-Duval et al. 2010). Furthermore, the high level of turbulence of $\sim 2.5 \mathrm{~km} \mathrm{~s}^{-1}$ present already at the beginning of the cloud evolution as well as the strongly fragmented sub-structure (Figure 2 indicate that the initial SN-driving period of 11.9 Myr was sufficient to establish a turbulent three-phase medium.

Considering the impact of external $\mathrm{SNe}$, we find that the cloud masses $M_{\mathrm{SN}}$ (bottom row of Figure 3) show only a moderate initial increase by $\sim 1 \%\left(\lesssim 1000 M_{\odot}\right)$ compared to $M_{\mathrm{noSN}}$, which we attribute to the compression of less dense gas by the incoming SN blast wave. We note that in a few runs, at later times, $M_{\mathrm{SN}}$ even slightly drops below $M_{\text {noSN }}$, i.e. the SN has a negative effect on the cloud mass, but the changes are always of the order of a few percent. The influence of the $\mathrm{SNe}$ on $\sigma$ (top row of Figure 3), however, is much more pronounced than on

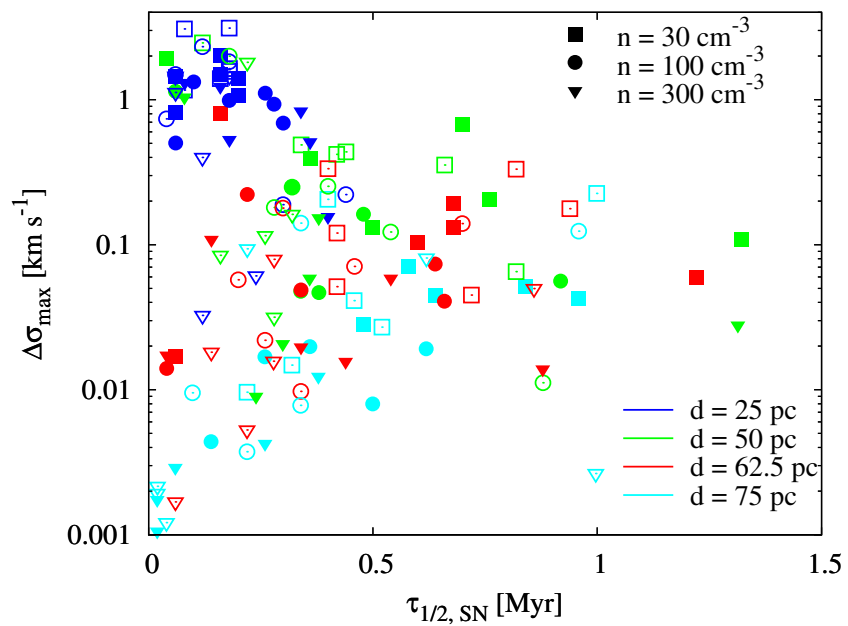

Figure 5. Relation between the decay time $\tau_{1 / 2, \mathrm{SN}}$ and the maximum increase $\Delta \sigma_{\max }$ of the additional turbulent velocity component caused by $\mathrm{SNe}$ with different distances (symbols) for $\mathrm{MC} 1$ and MC2 (open and closed symbols) calculated using different density thresholds (color-coded).

$M$. We find that in particular nearby $\mathrm{SNe}$ seem to have a noticeable effect on $\sigma$ increasing it by a few $\mathrm{km} \mathrm{s}^{-1}$. For more distant $(d>50 \mathrm{pc}) \mathrm{SNe}$, the impact is significantly lower.

In Figure 4 we show the relative change of $\sigma_{\mathrm{SN}}$ with respect to $\sigma_{\text {no SN }}$, now for all directions and the four distances considered in this work. Overall, the SN impact temporarily enhances $\sigma$ by at most $70 \%$ for the nearby $\mathrm{SNe}(d=25$ and $50 \mathrm{pc})$. For more distant $\mathrm{SNe}$, the relative increase is almost negligible with $\sim 1 \%$ for $d=$ $75 \mathrm{pc}$. Interestingly, even for nearby explosions, the increase is short-lived and the additional dispersion decays on timescales of a few $100 \mathrm{kyr}$.

\subsection{Maximum gain in velocity dispersion and decay time}

We determine the additional turbulent velocity dispersion driven by a nearby $\mathrm{SN}$,

$$
\Delta \sigma_{\max }=\max \left(\sigma_{\mathrm{SN}}-\sigma_{\mathrm{noSN}}\right)
$$

reached at $t=t_{\max }$, and its decay time (half-life), $\tau_{1 / 2, \mathrm{SN}}$, which is the time which passes until $\sigma_{\mathrm{SN}}-\sigma_{\text {no SN }}$ drops from $\Delta \sigma_{\max }$ to $0.5 \times \Delta \sigma_{\max }{ }^{1}$, i.e.

$$
\left(\sigma_{\mathrm{SN}}-\sigma_{\mathrm{noSN}}\right)\left(t_{\max }+\tau_{1 / 2, \mathrm{SN}}\right)=0.5 \times \Delta \sigma_{\max } .
$$

We do this for both MCs, but now using three different density thresholds of 30,100 , and $300 \mathrm{~cm}^{-3}$. Considering Figure 5. we find that with increasing distance, $\Delta \sigma_{\max }$ decreases. Furthermore, for $d \leq 50$ pc there seems to be a weak anti-correlation between $\tau_{1 / 2, \mathrm{SN}}$ and $\Delta \sigma_{\max }$ (and thus a positive correlation between $\tau_{1 / 2, \mathrm{SN}}$ and $d$ ). We attribute this to the fact that turbulence decays roughly within one crossing time, which is inversely proportional to $\sigma$ in the supersonic case (Stone et al. 1998). Furthermore, typically $\tau_{1 / 2, \mathrm{SN}} \sim 100-600 \mathrm{kyr}$, i.e. the effect of

\footnotetext{
1 Note that the half-life is related to the e-folding time $\tau_{\mathrm{e}}$ of an exponential decay as $\tau_{1 / 2, \mathrm{SN}}=\tau_{\mathrm{e}} \times \ln (2)$. When fitting an exponential function to the decaying part of $\sigma_{\mathrm{SN}}-\sigma_{\mathrm{no} \mathrm{SN}}$, we obtain very similar timescales. However, determining $\tau_{1 / 2, \mathrm{SN}}$ provides somewhat more robust estimates.
} 


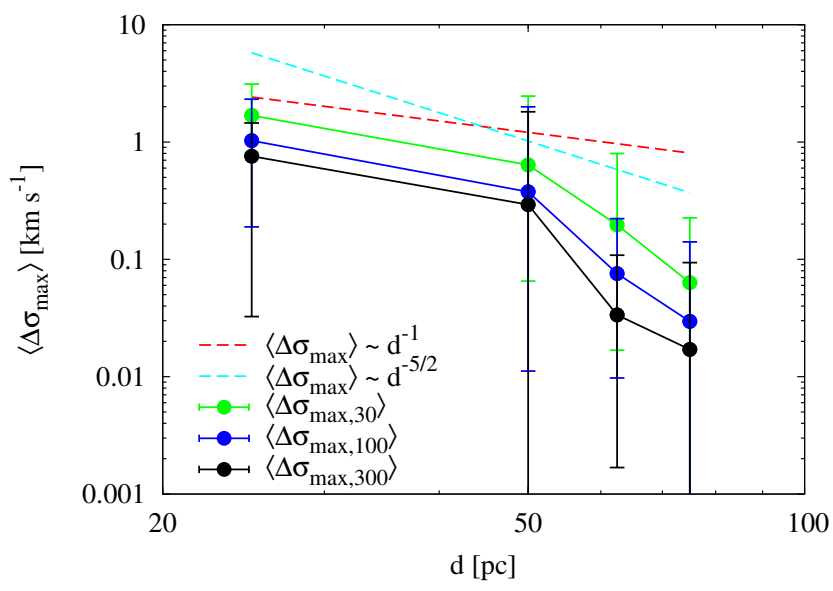

Figure 6. Mean maximum increase of the velocity dispersion $\left\langle\Delta \sigma_{\max }\right\rangle$ caused by $\mathrm{SNe}$ as a function of their distance averaged over MC1 and MC2. The error bar gives the minimum and maximum values found. For $d>50 \mathrm{pc}$, the velocity gain decreases steeper than expected for adiabatic expansion $\left(d^{-1}\right.$, red dashed line). For the momentum-conserving snowplow phase we would expect $\left\langle\Delta \sigma_{\max }\right\rangle \propto d^{-2.5}$ (cyan dashed line).

SNe is very limited in time compared to $\mathrm{MC}$ formation and evolution times of up to a few $10 \mathrm{Myr}$ (see e.g. the review by Dobbs et al. 2014). Since the SN energy is lost quickly by radiative cooling in the dense gas of the cloud (a few 1000 yr, see e.g Koyama \& Inutsuka 2000, Haid et al. 2016), $\sigma$ is only temporarily boosted and decays subsequently.

Combining the results of Figure 5 for $\mathrm{MC} 1$ and $\mathrm{MC} 2$, we calculate the mean value of $\Delta \sigma_{\max }$ for each $d$ (Figure 6). The error bars indicate the minimum and maximum value found. First, we see that $\left\langle\Delta \sigma_{\max }\right\rangle$ decreases with increasing density threshold. This is due to the dissipation of kinetic energy as the shock travels deeper into the cloud, i.e. into higher-density regions. Furthermore, for $d \leq 50 \mathrm{pc},\left\langle\Delta \sigma_{\max }\right\rangle$ is typically between a few $0.1-1$ $\mathrm{km} \mathrm{s}^{-1}$, whereas for $d=62.5 \mathrm{pc},\left\langle\Delta \sigma_{\max }\right\rangle \simeq 0.1 \mathrm{~km} \mathrm{~s}^{-1}$, i.e. less than the typical sound speed $c_{\mathrm{s}} \simeq 0.2 \mathrm{~km} \mathrm{~s}^{-1}$ of molecular gas with $T=10 \mathrm{~K}$. For $d=75 \mathrm{pc},\left\langle\Delta \sigma_{\max }\right\rangle$ only increases by a few $10 \mathrm{~m} \mathrm{~s}^{-1}$.

Assuming energy conservation, which is applicable if the $\mathrm{SN}$ is hitting the cloud during the Sedov-Taylor stage, we obtain the following for the energy gain of the cloud:

$$
\frac{1}{2} M_{\mathrm{MC}} \Delta \sigma_{\max }^{2}=\frac{A_{\mathrm{MC}}}{4 \pi d^{2}} \times E_{\mathrm{SN}} \times 0.27 .
$$

In the following we assume fiducial values of $A_{\mathrm{MC}}=\pi \times(10 \mathrm{pc})^{2} \quad$ and $\quad M_{\mathrm{MC}}=5 \times 10^{4} \mathrm{M}_{\odot} \quad$ for the area and mass of the MC, and take into account that only $27 \%$ of the SN energy $\left(E_{\mathrm{SN}}=10^{51} \mathrm{erg}\right)$ is in kinetic form during the Sedov-Taylor stage. This then results in a relation $\left\langle\Delta \sigma_{\max }\right\rangle \propto d^{-1}$ (red dashed line in Figure 6), which is similar to the observed slope for $d \leq$ $50 \mathrm{pc}$.

Furthermore, for the typical number densities of $0.2-$ $5 \mathrm{~cm}^{-3}$ of the regions, within which the $\mathrm{SNe}$ explode, the Sedov-Taylor radii are $r_{\mathrm{ST}} \simeq 5-35 \mathrm{pc}$ (Haid et al. 2016) and are always smaller than the distances of the SNe. Hence, the SN must have lost a significant amount of energy due to radiative cooling even before hitting the cloud. In particular for $d>50 \mathrm{pc}$, where the SN has entered the momentum-conserving snowplow phase, the decline of $\left\langle\Delta \sigma_{\max }\right\rangle$ is much steeper than $d^{-1}$. In this stage we expect an energy gain of the cloud of

$$
\frac{1}{2} M_{\mathrm{MC}} \Delta \sigma_{\max }^{2}=\frac{A_{\mathrm{MC}}}{4 \pi d^{2}} \times \frac{p_{\text {shell }}^{2}}{2 M_{\text {shell }}},
$$

assuming that all the mass in the volume occupied by the SN remnant is contained in the swept-up shell, i.e. $M_{\text {shell }} \propto d^{3}$, and that this swept-up shell has gained a momentum $p_{\text {shell }}$. This results in $\left\langle\Delta \sigma_{\max }\right\rangle \propto d^{-2.5}$ (cyan dashed line in Figure 6), which is closer to the decrease of $\left\langle\Delta \sigma_{\max }\right\rangle$ for $d>50 \mathrm{pc}$. However, at any $d$, the actual values for $\left\langle\Delta \sigma_{\max }\right\rangle$ are smaller than those of the two theoretical predictions, which indicates that the energy transfer from the SN shock to the MC is not very efficient (Walch \& Naab 2015).

It is difficult to find a common origin for the scatter of $\Delta \sigma_{\max }$ at a given distance. We do not see a correlation of $\Delta \sigma_{\max }$ with the density of the region in which the SN explodes, nor with the integrated column density along the path of the SN shock towards the center of the MC. It seems that $\Delta \sigma_{\max }$ is highly sensitive to the density distribution along the path of the shock (Haid et al. 2016) and can be significantly affected even by small Huctuations. This is also supported by the fact that the standard deviation of $\left\langle\Delta \sigma_{\max }\right\rangle$ increases with increasing distance, i.e. as there are more density fluctuations along the path of the SN shock. Moreover, differences in the cloud structure when seen from the 6 different directions also cause a scatter in $\Delta \sigma_{\max }$.

\subsection{The influence of magnetic fields}

In order to test whether magnetic fields measurably alter our results, we perform a corresponding simulation including magnetic fields. For this we use the same initial conditions as described in Section 2, but now include a magnetic field along the $x$-direction with a strength of

$$
B_{x}=B_{x, 0} \sqrt{\rho(z) / \rho_{0}},
$$

with $B_{x .0}=3 \mu \mathrm{G}$ in accordance with recent observations (e.g. Beck \& Wielebinski 2013). We insert SNe up to $t_{0, \text { mag }}=16 \mathrm{Myr}$ in order to establish a realistic multiphase ISM. We note that we here apply the SN driving for a longer time than for $\mathrm{MC} 1$ and $\mathrm{MC} 2$ since the magnetic field delays the formation of overdense structures, which subsequently form MCs (Walch et al. 2015, Girichidis et al. 2016, Girichidis et al., submitted). Afterwards, we follow the evolution of $2 \mathrm{MCs}$ - denoted as MC3 and MC4 - in selected regions with the same zoom-in strategy as before and insert a nearby $\mathrm{SN}^{2}(d=25,50$, or $62.5 \mathrm{pc})$ along 6 different directions at $t=t_{0, \mathrm{mag}}+1.5 \mathrm{Myr}$, i.e. once we have reached the final resolution of $0.12 \mathrm{pc}$.

We redo the same analysis as before by comparing the runs to a reference run without any nearby SN. A short overview of the results of these runs is given in the Appendix. Here, we only focus on the distance dependence of $\Delta \sigma_{\max }$ in Figure 7. Overall, we see a similar behaviour as for the runs without magnetic fields (Figure 6). The gain in $\sigma$ decreases with increasing $d$, in particular at $d>50 \mathrm{pc}$ the decrease becomes steeper. However, now

${ }^{2}$ Due to the small influence of SNe at $d=75 \mathrm{pc}$, we do not follow this case further here. 


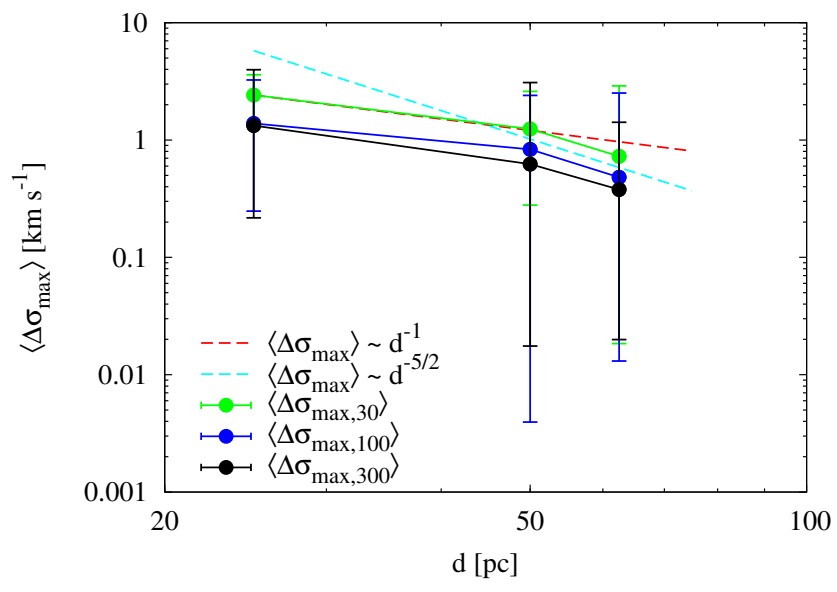

Figure 7. Same as in Figure 6 but now for the runs MC3 and MC4 including magnetic fields. Overall we see a similar behaviour as for the runs without magnetic fields, in particular the drop of $\left\langle\Delta \sigma_{\max }\right\rangle$ above $d=50 \mathrm{pc}$.

it is closer to the theoretical expectation of a $\mathrm{SN}$ in the momentum-conserving snowplow phase than for the unmagnetized runs. For $d \leq 50 \mathrm{pc}, \Delta \sigma_{\max }$ ranges from several $0.1 \mathrm{~km} \mathrm{~s}^{-1}$ and $\sim 3 \mathrm{~km} \mathrm{~s}^{-1}$, whereas for $d=62.5 \mathrm{pc}$ it drops below $0.7 \mathrm{~km} \mathrm{~s}^{-1}$.

The induced level of turbulence is thus somewhat higher compared to the non-magnetic runs (a factor of $\sim 2$ ). On the one hand, this could indicate that magnetic fields support the transport of turbulence from the diffuse into the dense ISM. On the other hand, as we will show in Section 3.5, this can partly be attributed to the fact that we model the SN impact at a stage where the clouds are in a relatively early assembly phase with masses of $\sim$ $1.5 \times 10^{4} \mathrm{M}_{\odot}$ only (bottom left panel of Fig. 11 , i.e. significantly less massive than the MCs without magnetic fields at this stage $\left(4.1\right.$ and $2.4 \times 10^{4} \mathrm{M}_{\odot}$ for MC1 and $\mathrm{MC} 2$, respectively). However, overall our main findings of a moderate influence of external $\mathrm{SNe}$ on MCs seem to also hold in the presence of magnetic fields.

\subsection{SN impact at a later stage}

As can be seen from the mass evolution of MC1 and MC2 (bottom row of Figure 3), the SNe which go off at $t_{\mathrm{SN}}=t_{0}+1.5 \mathrm{Myr}$ impact the clouds during their assembly phase, which is completed around $t=t_{0}+2 \mathrm{Myr}$. Similar also applies to the magnetized runs MC3 and MC4 (Figure 11). In order to test possible differences when a cloud is hit by an SN at a later stage of its evolution, we repeat the previously discussed simulations but now with SNe going off at $t_{\mathrm{SN}}=t_{0}+2.5 \mathrm{Myr}$ and $t_{0, \operatorname{mag}}+2.5 \mathrm{Myr}$ for the case without and with magnetic fields, respectively. Since we found that for distances larger than $50 \mathrm{pc}$ the influence is almost negligible (Figures 6 and 7), we only consider the cases $d=25$ and 50 pc.

We repeat the previously described analysis and determine $\Delta \sigma_{\max }$ and $\tau_{1 / 2, \mathrm{SN}}$ (Equations 2 and 3). In Figure 8 we plot the ratio of the half-life times determined for the SNe going off at the two different times (2.5 and 1.5 Myr) against the ratio of $\Delta \sigma_{\max }$ using the same symbols as in Figure 5. The scatter in the ratios seems to be similar for all four clouds considered. Furthermore, we find that for both $d$ the scatter in the distribution is somewhat larger for higher MC density thresholds. We argue that this is a consequence of the shorter dynamical timescales in higher-density regions which allow for more variations in these regions within $1 \mathrm{Myr}$. For the decay time of the initial gain in velocity, we do not see a clear trend here.

Concerning the strength of the impact, however, SNe going off at later stages seem to have - on average - a slightly reduced influence $\left(\Delta \sigma_{\max }(2.5 \mathrm{Myr}) / \Delta \sigma_{\max }(1.5 \mathrm{Myr})<1\right) . \quad$ We speculate that this is due to the fact that, as the cloud evolves, its density contrast increases: the already dense regions become even denser and are thus harder to affect with incoming SN shocks. Furthermore, larger low-density regions might evolve due to the accretion of material on the denser regions, which might allow the SN blast to pass relatively unhindered. Finally, at $t_{\mathrm{SN}}=t_{0}+2.5 \mathrm{Myr}\left(t_{\mathrm{SN}}=t_{0, \mathrm{mag}}+2.5 \mathrm{Myr}\right.$ for the runs with magnetic fields, respectively), also the total masses of the clouds have increased by about $30-40 \%$ (up to a maximum of $5.5 \times 10^{4} \mathrm{M}_{\odot}$ for $\mathrm{MC} 1$ ), which also might reduce the influence of an incoming shock carrying a fixed amount of momentum.

\section{DO SUPERNOVAE DRIVE MOLECULAR CLOUD TURBULENCE?}

In order to assess whether many SNe exploding in the vicinity of an $\mathrm{MC}$ can maintain its turbulent state, we estimate the rate of $\mathrm{SNe},(\mathrm{d} \mathrm{SNe} / \mathrm{d} t)_{\text {impact, }}$, which could significantly impact the cloud velocity dispersion. Based on our results, we only consider SNe with $d \leq 50 \mathrm{pc}$ (Figures 6 and 7). Applying the Kennicutt-Schmidt relation, we can link the star formation rate to the gas surface density via $\Sigma_{\text {SFR }} \propto \Sigma_{\text {gas }}^{1.4}$ (Equation 4 of Kennicutt 1998). By assuming a standard IMF (Chabrier 2001), which results in 1 SN per $100 \mathrm{M}_{\odot}$ of stars, we then convert $\Sigma_{\mathrm{SFR}}$ into an SN rate, $\Sigma_{\mathrm{SNR}}$. This leads to $15 \mathrm{SNe} \mathrm{Myr}^{-1}(500 \mathrm{pc})^{-2}$ for $\Sigma_{\text {gas }}=10 \mathrm{M}_{\odot} \mathrm{pc}^{-2}$ (see Walch et al. 2015, Girichidis et al. 2016). With this we obtain the relation

$$
\begin{aligned}
\left(\frac{\mathrm{d} \mathrm{SNe}}{\mathrm{d} t}\right)_{\text {impact }} & =\Sigma_{\mathrm{SNR}} \times \pi \times(50 \mathrm{pc})^{2} \\
& \simeq 0.14 \frac{\mathrm{SNe}}{300 \mathrm{kyr}}\left(\frac{\Sigma_{\text {gas }}}{10 \mathrm{M}_{\odot} \mathrm{pc}^{-2}}\right)^{1.4},
\end{aligned}
$$

where $\left(\frac{\mathrm{d} S N e}{\mathrm{~d} t}\right)_{\text {impact }}$ is the number of SNe that hit a cloud in a given time interval under the assumption of random SN locations.

Since we found a typical decay time $\tau_{1 / 2, \mathrm{SN}} \simeq 300_{-200}^{+300}$ kyr for the externally driven turbulent component in an MC (see Figure 5 and right panel of Figure 11), an MC has to be hit by one SN per $300_{-200}^{+300} \mathrm{kyr}$ to maintain the observed level of MC turbulence of a few $1 \mathrm{~km} \mathrm{~s}^{-1}$ (Larson 1981; Solomon et al. 1987; Elmegreen \& Falgarone 1996; Heyer et al. 2001; Roman-Duval et al. 2010), which happens for $\Sigma_{\text {gas }} \gtrsim 40_{-15}^{+49} \mathrm{M}_{\odot} \mathrm{pc}^{-2}$. We note that this estimate assumes that SNe in environments with higher $\Sigma_{\text {gas }}$ are as effective as found in this study, which is not necessarily the case ( $\mathrm{Li}$ et al. 2017).

However, for solar neighbourhood conditions $\left(\Sigma_{\text {gas }}=\right.$ $10 \mathrm{M}_{\odot} \mathrm{pc}^{-2}$ ), an $\mathrm{MC}$ is hit by a nearby $\mathrm{SN}$ once per $\sim 2.1$ Myr. In the time interval between two hits, the initial gain in $\sigma$ thus decreases to $\sim 1 \%$ of its original 
$\mathrm{MC} 1+\mathrm{MC} 2$, no B-field

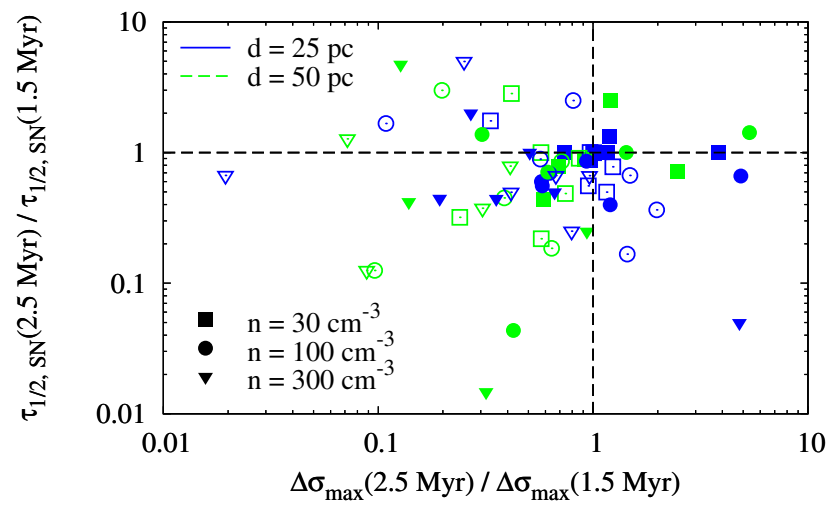

MC3 + MC4, with B-field

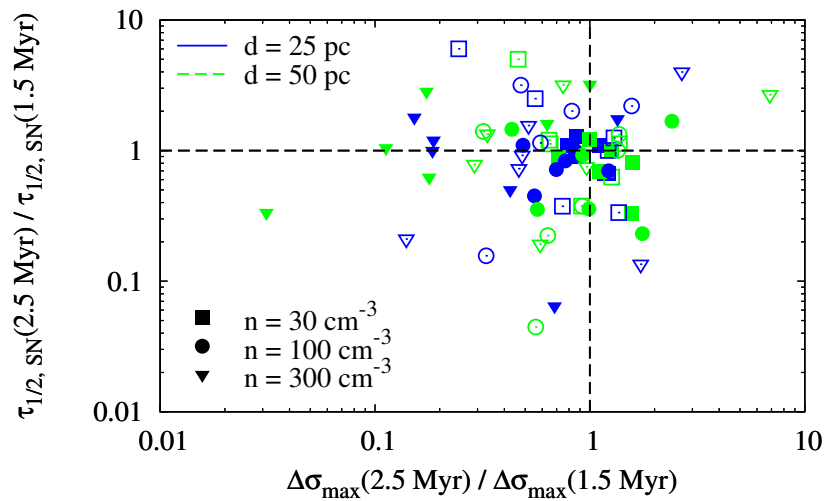

Figure 8. Ratio of the half-life at $2.5 \mathrm{Myr}$ and $1.5 \mathrm{Myr}$ against the corresponding ratio of $\Delta \sigma_{\max }$ for MC1 (filled symbols) and MC2 (open symbols) without magnetic fields (left panel) and MC3 (filled symbols) and MC4 (open symbols) with magnetic fields (right panel). At the later stage, on average the strength of the SN impact is reduced.

value. Hence, even for the nearest $\mathrm{SNe}(d=25 \mathrm{pc}$, see Figure 5), the increase of the turbulent velocity after $2.1 \mathrm{Myr}$ is $\lesssim 10 \mathrm{~m} \mathrm{~s}^{-1}$, i.e. significantly lower than the velocity dispersion of a few $\mathrm{km} \mathrm{s}^{-1}$ found in Galactic MCs with masses comparable to those of the clouds presented here (e.g. Larson 1981; Solomon et al. 1987; Roman-Duval et al. 2010). Therefore, if $\sigma$ were to be maintained by external SNe explosions alone, this would result in significantly lower values than observed.

On the other hand, in the Galactic Central Molecular Zone (CMZ, typical radius $\sim 250 \mathrm{pc}$ ), SN rates of about $1 \mathrm{SN}$ per $1 \mathrm{kyr}$ are reported (e.g. Crocker et al. 2011, Crocker 2012 Ponti et al. 2015), which translates to $\left(\frac{\mathrm{d} S \mathrm{Ne}}{\mathrm{d} t}\right)_{\text {impact }} \simeq 10 \mathrm{SN}$ per $300 \mathrm{kyr}$. Based on our study, this rate would be high enough to sustain the MC turbulence by SN explosions. This is in line with recent results by Kauffmann et al. (2017), who found a steep linewidth-size relation in MCs in the Galactic Center, which indicates shock-dominated turbulent motions as e.g. created by SNe.

We argue that - as we find a comparable effect of SNe for magnetized and unmagnetized clouds (Section 3.4) as well as at later times (Section 3.5. here the influence seems to be even slightly reduced) - the results above should hold for a wide range of evolutionary stages and physical conditions of MCs.

\subsection{Gravity or SN-driven turbulence?}

In Figure 9, we show the gas velocity dispersion in MC1 as a function of density at four different times. Compared to the sound speed, which corresponds to the equilibrium temperature at a given density (cyan line), the mean velocity dispersion in the cloud (black line) is highly supersonic in gas with $\rho \gtrsim 10^{-24} \mathrm{~g} \mathrm{~cm}^{-3}$. This could be partly attributed to accretion-driven turbulence (Klessen \& Hennebelle 2010, Goldbaum et al. 2011). At $\rho \gtrsim 10^{-20} \mathrm{~g} \mathrm{~cm}^{-3}$, the velocity dispersion even increases with density, which is a sign for local gravitational collapse.

The latter result is in line with recent simulations by Ibáñez-Mejía et al. (2016, 2017) and observations by Heyer et al. (2009), who claimed that gravity is the main driver of turbulence in MCs. Our results also agree with
Iffrig \& Hennebelle (2015), who found that the influence of SNe located outside MCs is only moderate and temporally limited to a few $100 \mathrm{kyr}$. However, the authors used a highly idealized setup and limit the SN distance to at most $7 \mathrm{pc}$, which is why a proper comparison with our work is difficult.

We emphasize that our results are still in agreement with the scenario in which MCs form from gas that is swept-up by SN explosions (Koyama \& Inutsuka 2000, Inoue \& Inutsuka 2009; Inutsuka et al. 2015). In our simulations, cloud formation is mainly triggered by $\mathrm{SNe}$ going off prior to $t_{0}$, which sweep up turbulent gas while their shocks travel through the three-phase ISM.

However, at first glance our results seem to be in an apparent contradiction to Padoan et al. (2016), who found that SNe alone can maintain turbulence in MCs using turbulent box simulations with a comparable resolution of 0.24 pc. They used a rate of $\Sigma_{\mathrm{SNR}}=25 \mathrm{SNe} \mathrm{Myr}^{-1}(500 \mathrm{pc})^{-2}$, which gives $\left(\frac{\mathrm{dSNe}}{\mathrm{d} t}\right)_{\text {impact }}=0.24 \mathrm{SNe}$ per $300 \mathrm{kyr}$ (Equation 7 ) and would therefore be too low to drive MC turbulence in a stratified environment. There are, however, significant differences in our setup. Padoan et al. (2016) used periodic boundary conditions, which could enhance the impact of SN explosions compared to a stratified disc setup (compare Gatto et al. 2015, with Girichidis et al. 2016), as well as a different parameterized cooling function without a chemical network. Furthermore, due to the random distribution of $\mathrm{SNe}$ in their simulation domain, a small fraction of SNe might also go off in the clouds themselves, which we do not consider here. Also the different usage of self-gravity can contribute to the differences: whereas Padoan et al. (2016) turned on gravity after an initial driving phase of several $10 \mathrm{Myr}$, in our case gravity is applied from the start of the simulations. In a recent follow-up work, Padoan et al. (2017) extended their study to even higher resolutions of 0.0076 pc. However, since the authors only focused on the state of their clouds after self-gravity is switched on, a direct comparison to our results is difficult.

In this context, we note that we have recently investigated the propagation of turbulence from the diffuse into the dense ISM by means of idealized simulations with a resolution of $0.0078 \mathrm{pc}$. We find that the trans- 


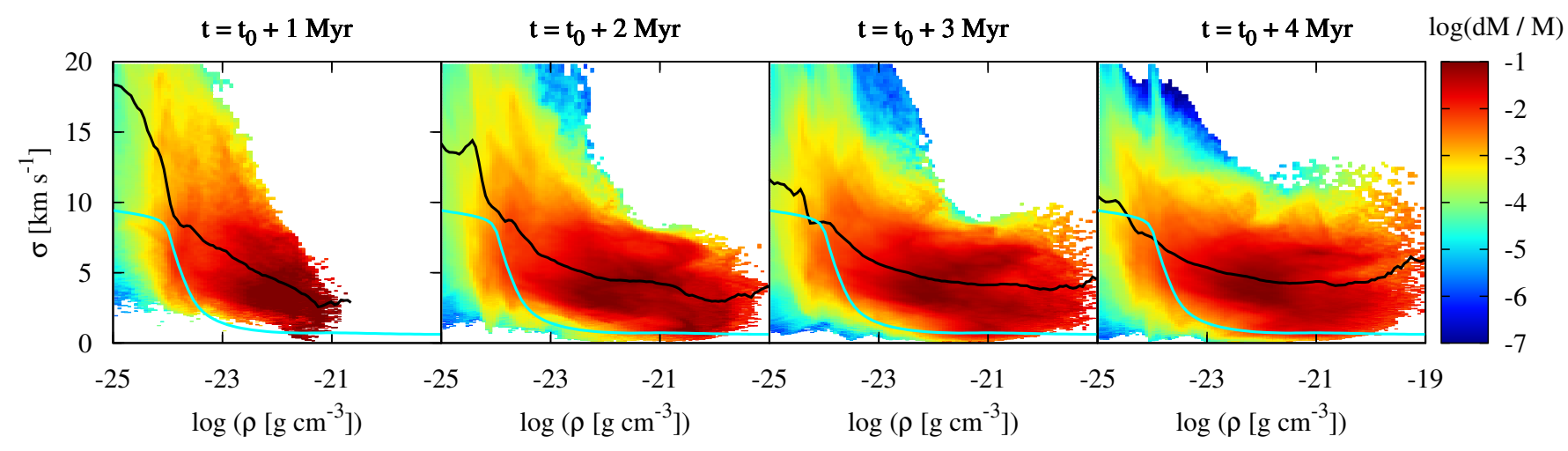

Figure 9. Time evolution of the density- $\sigma$-distribution for MC1. For densities above $\sim 10^{-24} \mathrm{~g} \mathrm{~cm}^{-3}$ the mean $\sigma$ in each density bin (black line) is clearly supersonic when compared to the equilibrium sound speed (cyan line) and, at late times, even starts to increase towards the highest densities.

port of turbulence from the low-density environment into the dense medium is inefficient (Rathjen et al. 2018, in preparation), which is in line with our results presented here.

However, we emphasize that, since Padoan et al. (2016) did not include self-gravity initially, their clouds might be more diffuse and thus are probably in an earlier evolutionary stage than ours at the time we inject the SNe. As stated before, at this earlier stage, the turbulence of our $\mathrm{MCs}$ is mainly generated by SNe, which is in agreement with the results of Padoan et al. (2016). Only subsequently, as the clouds become more massive and denser, the importance of external SNe reduces (Section 3.5) and gravity seems to become mainly responsible for maintaining the turbulent stage of MCs. To summarize, our work suggests that - depending on the evolutionary stage of the cloud - different turbulence drivers might be at work.

\section{CONCLUSIONS}

We present simulations of MC formation within their galactic environment with an effective resolution of $0.1 \mathrm{pc}$ including the chemical evolution of the clouds. Our MCs inherit the initial level of turbulence from the diffuse gas they formed in. After they have condensed out of the diffuse ISM, we systematically explore the effect of individual SNe going off outside the clouds at different distances.

Nearby SNe $(\mathrm{d} \sim 25 \mathrm{pc})$ boost the turbulent velocity dispersions by up to a few $1 \mathrm{~km} \mathrm{~s}^{-1}$, whereas for more distant $\mathrm{SNe}(d>50 \mathrm{pc})$ the boost is almost negligible. Independent of the distance, however, at these later stages the SNe exploding outside the clouds can increase the level of turbulence only temporarily for a few $100 \mathrm{kyr}$. Furthermore, we find that the later the SN goes off, the lower is the influence of the SN on the cloud. This holds for MCs with masses from $\sim 1$ to $5.5 \times 10^{4} \mathrm{M}_{\odot}$ and MCs with and without magnetic fields. Furthermore, we tentatively argue that magnetic fields slightly support the transport of turbulence from the diffuse ISM into the dense clouds.

With these results, the following picture for MC formation under solar neighbourhood conditions emerges: in the earliest phase of MC formation, SNe can play a significant role in seeding the initial level of turbulence. At later stages, however, nearby $\mathrm{SNe}$ are not able to maintain the turbulent state of MCs. We argue that here turbulence has to be maintained rather by turbulent gas accretion, the onset of local gravitational collapse, and internal stellar feedback. However, in galactic environments with gas surface densities above $40 \mathrm{M}_{\odot} \mathrm{pc}^{-2}$, we expect $\mathrm{SNe}$ to be able to sustain MC turbulence. In particular in the Galactic CMZ, cloud turbulence driven by SN shocks could be present.

\section{ACKNOWLEDGEMENTS}

The authors like to thank the anonymous referee for the comments which helped to significantly improve the paper. DS and SW acknowledge the support of the BonnCologne Graduate School, which is funded through the German Excellence Initiative. DS and SW also acknowledge funding by the Deutsche Forschungsgemeinschaft (DFG) via the Sonderforschungsbereich SFB 956 Conditions and Impact of Star Formation (subproject C5). SW and SH acknowledge support via the ERC starting grant No. 679852 "RADFEEDBACK". SW, and TN acknowledge support from the DFG Priority Program 1573 "Physics of the Interstellar Medium". PG acknowledges funding from the European Research Council under ERC-CoG grant CRAGSMAN-646955. TN acknowledges support from the DFG cluster of excellence "Origin and Structure of the Universe". The FLASH code used in this work was partly developed by the Flash Center for Computational Science at the University of Chicago. The simulations were performed at SuperMUC at the Leibniz-Rechenzentrum Garching.

\section{REFERENCES}

Ballesteros-Paredes, J., Klessen, R. S., Mac Low, M.-M., \&

Vazquez-Semadeni, E. 2007, Protostars and Planets V, 63

Beck, R., \& Wielebinski, R. 2013, Magnetic Fields in Galaxies, ed. T. D. Oswalt \& G. Gilmore, 641

Blondin, J. M., Wright, E. B., Borkowski, K. J., \& Reynolds, S. P. 1998, ApJ, 500, 342

Bouchut, F., Klingenberg, C., \& Waagan, K. 2007, Numerische Mathematik, 108, 7, 10.1007/s00211-007-0108-8.

http://dx.doi.org/10.1007/s00211-007-0108-8

Chabrier, G. 2001, ApJ, 554, 1274

Clark, P. C., Glover, S. C. O., \& Klessen, R. S. 2012, MNRAS, 420,745

Crocker, R. M. 2012, MNRAS, 423, 3512

Crocker, R. M., Jones, D. I., Aharonian, F., et al. 2011, MNRAS, 413, 763

Dobbs, C. L., Krumholz, M. R., Ballesteros-Paredes, J., et al. 2014, Protostars and Planets VI, 3 

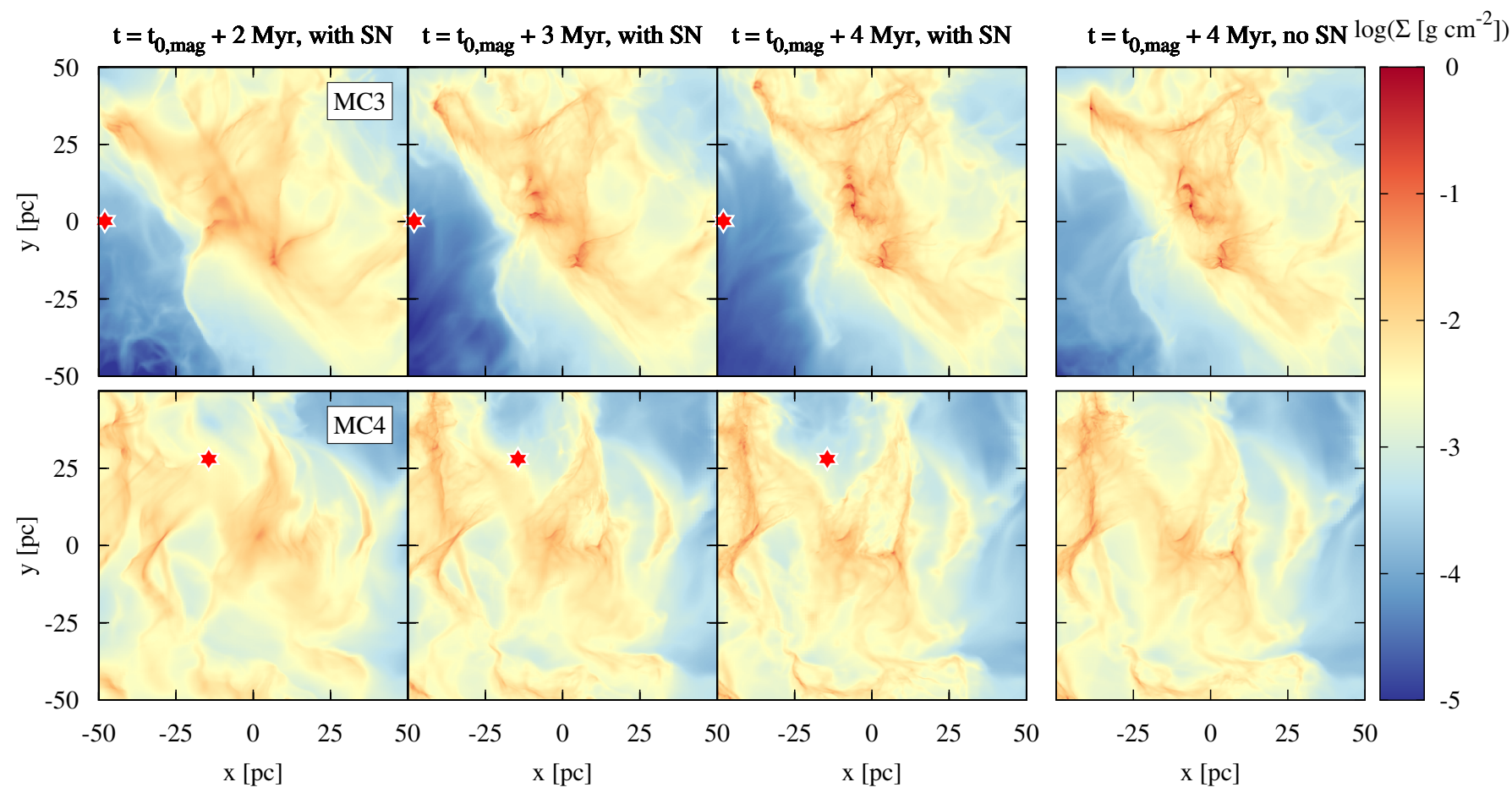

Figure 10. Time evolution of the column density of the runs MC3 and MC4 with magnetic fields exposed to a nearby SN at a distance of $50 \mathrm{pc}$ and $25 \mathrm{pc}$ for MC3 and MC4, respectively. As for unmagnetized runs, mainly the low-density regions are affected. For comparison, the right panels show the reference run without any SN.

Draine, B. T. 1978, ApJS, 36, 595

Dubey, A., Fisher, R., Graziani, C., et al. 2008, in Astronomical Society of the Pacific Conference Series, Vol. 385, Numerical Modeling of Space Plasma Flows, ed. N. V. Pogorelov, E. Audit, \& G. P. Zank, 145

Elmegreen, B. G., \& Falgarone, E. 1996, ApJ, 471, 816

Elmegreen, B. G., \& Scalo, J. 2004, ARA\&A, 42, 211

Fryxell, B., Olson, K., Ricker, P., et al. 2000, ApJS, 131, 273

Gatto, A., Walch, S., Mac Low, M.-M., et al. 2015, MNRAS, 449, 1057

Girichidis, P., Walch, S., Naab, T., et al. 2016, MNRAS, 456, 3432

Glover, S. C. O., Federrath, C., Mac Low, M.-M., \& Klessen, R. S. 2010, MNRAS, 404, 2

Glover, S. C. O., \& Mac Low, M.-M. 2007, ApJ, 659, 1317

Goldbaum, N. J., Krumholz, M. R., Matzner, C. D., \& McKee, C. F. 2011, ApJ, 738, 101

Habing, H. J. 1968, Bull. Astron. Inst. Netherlands, 19, 421

Haid, S., Walch, S., Naab, T., et al. 2016, MNRAS, 460, 2962

Hewitt, J. W., \& Yusef-Zadeh, F. 2009, ApJL, 694, L16

Heyer, M., Krawczyk, C., Duval, J., \& Jackson, J. M. 2009, ApJ, 699, 1092

Heyer, M. H., Carpenter, J. M., \& Snell, R. L. 2001, ApJ, 551, 852

Hirota, A., Kuno, N., Sato, N., et al. 2011, ApJ, 737, 40

Ibáñez-Mejía, J. C., Mac Low, M.-M., Klessen, R. S., \& Baczynski, C. 2016, ApJ, 824, 41

—. 2017, ArXiv e-prints, arXiv:1705.01779

Iffrig, O., \& Hennebelle, P. 2015, A\&A, 576, A95

Inoue, T., \& Inutsuka, S.-i. 2009, ApJ, 704, 161

Inutsuka, S.-i., Inoue, T., Iwasaki, K., \& Hosokawa, T. 2015, A\&A, 580, A49

Kauffmann, J., Pillai, T., Zhang, Q., et al. 2017, A\&A, 603, A89

Kennicutt, Jr., R. C. 1998, ApJ, 498, 541

Klessen, R. S., \& Hennebelle, P. 2010, A\&A, 520, A17

Koyama, H., \& Inutsuka, S.-I. 2000, ApJ, 532, 980

Larson, R. B. 1981, MNRAS, 194, 809
Li, M., Bryan, G. L., \& Ostriker, J. P. 2017, ApJ, 841, 101 Mac Low, M.-M., \& Klessen, R. S. 2004, Reviews of Modern Physics, 76, 125

Mac Low, M.-M., Klessen, R. S., Burkert, A., \& Smith, M. D. 1998, Physical Review Letters, 80, 2754

Miville-Deschênes, M.-A., Murray, N., \& Lee, E. J. 2017, ApJ, 834,57

Naab, T., \& Ostriker, J. P. 2017, ARA\&A, 55, 59

Nelson, R. P., \& Langer, W. D. 1997, ApJ, 482, 796

Neufeld, D. A., Hollenbach, D. J., Kaufman, M. J., et al. 2007, ApJ, 664, 890

Padoan, P., Haugbølle, T., Nordlund, Å., \& Frimann, S. 2017, ApJ, 840, 48

Padoan, P., Pan, L., Haugbølle, T., \& Nordlund, Å. 2016, ApJ, 822, 11

Ponti, G., Morris, M. R., Terrier, R., et al. 2015, MNRAS, 453, 172

Roman-Duval, J., Jackson, J. M., Heyer, M., Rathborne, J., \& Simon, R. 2010, ApJ, 723, 492

Seifried, D., Walch, S., Girichidis, P., et al. 2017, MNRAS, 472, 4797

Solomon, P. M., Rivolo, A. R., Barrett, J., \& Yahil, A. 1987, ApJ, 319,730

Stone, J. M., Ostriker, E. C., \& Gammie, C. F. 1998, ApJL, 508, L99

Tang, X., \& Chevalier, R. A. 2014, ApJL, 784, L35

Waagan, K. 2009, Journal of Computational Physics, 228, 8609 . http://www.sciencedirect.com/science/article/ B6WHY-4X3W4C6-1/2/e979801e0d50aa4810c5cb3d1edd36a9 Walch, S., \& Naab, T. 2015, MNRAS, 451, 2757

Walch, S., Girichidis, P., Naab, T., et al. 2015, MNRAS, 454, 238

Williams, J. P., de Geus, E. J., \& Blitz, L. 1994, ApJ, 428, 693

Wünsch, R., Walch, S., Whitworth, A. P., \& Dinnbier, F. 2017, ArXiv e-prints, arXiv:1708.06142 

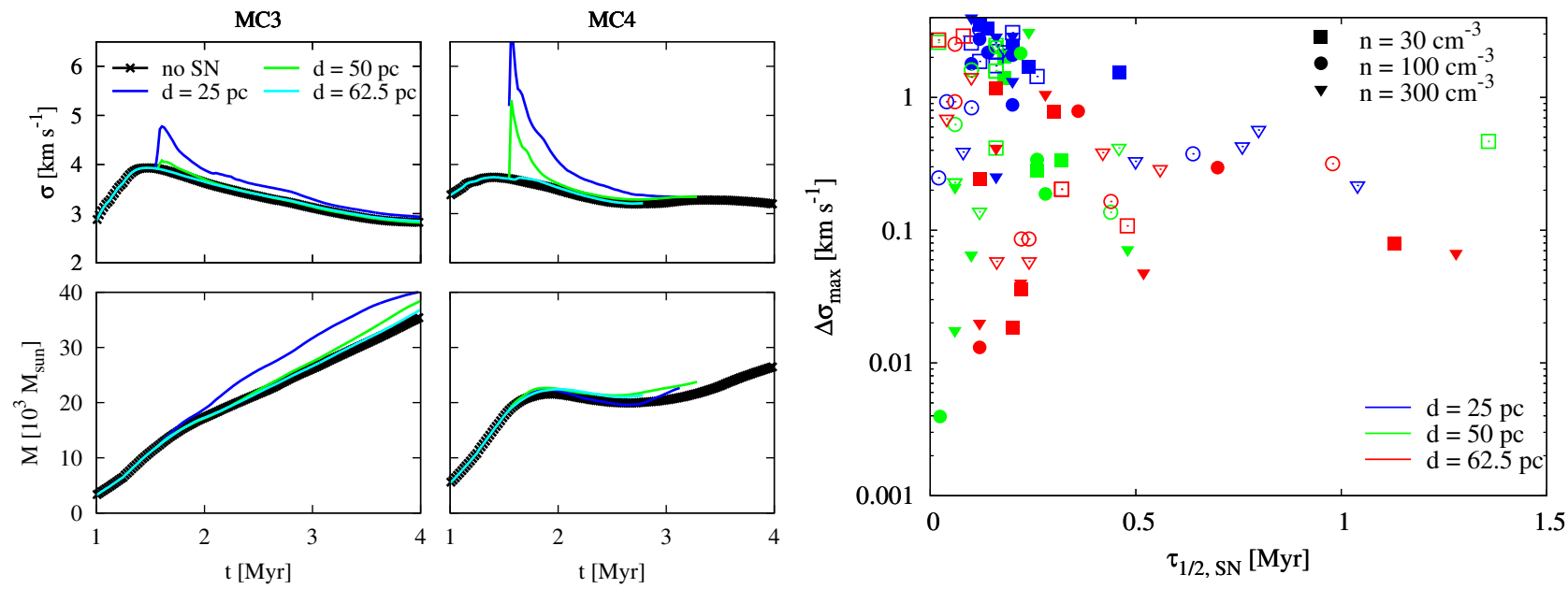

Figure 11. Left: Same as in Figure 3 but not for the runs MC3 and MC4 with magnetic fields. Right: Same as in Figure 5 but for the runs MC3 and MC4. Also for runs including magnetic fields, the effect of SNe seems to be temporally limited to a few 100 kyr. The mass of the clouds is only marginally affected.

\section{APPENDIX}

\section{INFLUENCE OF MAGNETIC FIELDS}

In Figure 10 we show the time evolution of the column density of the runs MC3 and MC4 including magnetic fields. Similar to MC1 and MC2, the nearby SNe seem to mainly affect the lower-density regions of the clouds.

In Figure 11 we show the evolution of the mass and velocity dispersion of gas with $n \geq 100 \mathrm{~cm}^{-3}$. As for MC1 and $\mathrm{MC} 2$, the mass of MC4 remains rather constant at $t=t_{0, \mathrm{mag}}+2 \mathrm{Myr}$ with around $2.5 \times 10^{4} \mathrm{M}_{\odot}$, whereas for $\mathrm{MC} 3$ it increases steadily over time to $\sim 3.5 \times 10^{4} \mathrm{M}_{\odot}$. However, considering the effect of a nearby SN, for both clouds $M_{\mathrm{SN}}$ shows only a marginal increase of $\lesssim 10 \%$, similar to the runs without magnetic fields. We note that due to computational cost reasons, we did not run all simulations for $4 \mathrm{Myr}$, but stopped them when the additional velocity gain has dropped to almost zero compared to the reference run.

The velocity dispersion (top row in the left panel of Figure 11) of the unperturbed clouds (black line with crosses) quickly reaches a roughly constant level of $\sim 3-4 \mathrm{~km} \mathrm{~s}^{-1}$ for both MCs. Considering the impact of nearby SNe, we find behaviour very similar to that of the unmagnetized runs: once the SN blast wave impacts the cloud, it experiences an increase which is significantly more pronounced for $d \leq 50 \mathrm{pc}$ and subsequently decays within a few 100 kyr.

This is also reflected in the right panel of Figure 11, where for each run we show $\Delta \sigma_{\max }$ and $\tau_{1 / 2, \mathrm{SN}}$, following the approach described in Section 3.3. As for the unmagnetized runs, we find typical values of $\tau_{1 / 2, \mathrm{SN}}$ of a few $100 \mathrm{kyr}$ and - depending on $d$ - values of $\Delta \sigma_{\max }$ ranging from $\sim 10 \mathrm{~m} \mathrm{~s}^{-1}$ up to a few $1 \mathrm{~km} \mathrm{~s}^{-1}$.

To summarize, overall the runs with magnetic fields thus show behaviors qualitatively and quantitatively similar to those of the runs without magnetic fields. 